Clinical

\title{
Five-Year Outcomes With Biodegradable-Polymer Sirolimus-Eluting Stents Versus Durable-Polymer Everolimus-Eluting Stents in Patients With Acute Coronary Syndrome: A Subgroup Analysis of the BIOSCIENCE Trial
}

\author{
Juan F. Iglesias ${ }^{\mathrm{a}}$, Dik Heg ${ }^{\mathrm{b}}$, Marco Roffi ${ }^{\mathrm{a}}$, Sophie Degrauwe ${ }^{\mathrm{a}}$, David Tüller ${ }^{\mathrm{c}}$, Olivier Muller ${ }^{\mathrm{d}}$, Miriam Brinkert ${ }^{\mathrm{e}}$, \\ Stéphane Cook ${ }^{\mathrm{f}}$, Daniel Weilenmann ${ }^{\mathrm{g}}$, Christoph Kaiser ${ }^{\mathrm{h}}$, Florim Cuculi ${ }^{\mathrm{i}}$, Marco Valgimigli ${ }^{\mathrm{j}}$, Peter Jüni ${ }^{\mathrm{k}}$, \\ Stephan Windecker ${ }^{\mathrm{j}}$, Thomas Pilgrim ${ }^{\mathrm{j}, *}$ \\ a Department of Cardiology, Geneva University Hospitals, Geneva, Switzerland \\ ${ }^{\mathrm{b}}$ CTU Bern, University of Bern, Bern, Switzerland \\ c Department of Cardiology, Triemlispital, Zurich, Switzerland \\ d Department of Cardiology, Lausanne University Hospital, Lausanne, Switzerland \\ e Department of Cardiology, Kantonsspital Aarau, Aarau, Switzerland \\ ${ }^{\mathrm{f}}$ Department of Cardiology, University and Hospital Fribourg, Fribourg, Switzerland \\ g Department of Cardiology, Kantonsspital St Gallen, St Gallen, Switzerland \\ h Department of Cardiology, Basel University Hospital, Basel, Switzerland \\ i Department of Cardiology, Kantonsspital Lucerne, Lucerne, Switzerland \\ ${ }^{\mathrm{j}}$ Department of Cardiology, Inselspital, University of Bern, Bern University Hospital, Bern, Switzerland

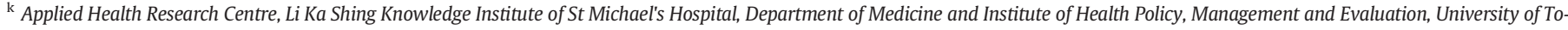 \\ ronto, Toronto, Canada
}

\section{A R T I C L E I N F O}

\section{Article history:}

Received 2 September 2020

Received in revised form 3 February 2021

Accepted 5 February 2021

\section{Keywords:}

Acute coronary syndrome

Biodegradable polymer

Drug-eluting stent

Clinical outcomes

\begin{abstract}
A B S T R A C T
Background: Thin-strut biodegradable polymer sirolimus-eluting stents (BP-SES) have been shown to reduce target lesion failure (TLF) at one-year follow-up compared with durable polymer everolimus-eluting stents (DPEES) among patients with acute coronary syndrome (ACS). The long-term clinical benefits of thin-strut BP-SES over DP-EES in ACS patients after complete degradation of the polymer coating remain uncertain. Methods: We performed a post-hoc subgroup analysis of ACS patients included into the BIOSCIENCE randomized trial (NCT01443104). The primary endpoint was target lesion failure (TLF), a composite of cardiac death, targetvessel myocardial infarction or clinically indicated target lesion revascularization, at 5 years.

Results: Among 2119 patients enrolled between March 2012 and May 2013, 1131 (53\%) presented with ACS. The 5 -year cumulative incidence of TLF was significantly lower in patients with ACS compared to chronic coronary syndrome [ $16.5 \%$ vs. $22.9 \%$; rate ratio (RR), $0.69 ; 95 \%$ confidence interval (CI), $0.57-0.85 ; p<0.001$ ]. At 5 years, TLF occurred similarly in ACS patients treated with BP-SES and DP-EES (16.9\% vs. 16.0\%; RR, 1.04; 95\% $\mathrm{CI}, 0.78-1.41 ; p=0.78$ ). The individual components of the primary endpoint did not differ between ACS patients treated with BP-SES or DP-EES at 5 years. Overall, there was no interaction between clinical presentation and treatment effect.

Conclusions: In a subgroup analysis of the BIOSCIENCE trial, we found no difference in long-term outcomes between ACS patients treated with BP-SES or DP-EES at 5 years.
\end{abstract}

(C) 2021 The Authors. Published by Elsevier B.V. This is an open access article under the CC BY-NC-ND license (http://creativecommons.org/licenses/by-nc-nd/4.0/).

\footnotetext{
Abbreviations: ACS, acute coronary syndrome;; BP-SES, biodegradable polymer sirolimus-eluting stents;; CCS, chronic coronary syndrome;; CI, confidence interval;; DES, drug-eluting stent;; DP-EES, durable polymer everolimus-eluting stents;; MI, myocardial infarction;; PCI, percutaneous coronary intervention;; RR, rate ratio;; STEMI, STsegment elevation myocardial infarction;; TLF, target lesion failure;; TLR, target lesion revascularization.

* Corresponding author at: Department of Cardiology, Inselspital, Bern University Hospital, University of Bern, Freiburgstrasse 18, 3010 Bern, Switzerland.

E-mail address: Thomas.Pilgrim@insel.ch (T. Pilgrim).
}

\section{Introduction}

Percutaneous coronary intervention $(\mathrm{PCI})$ is the preferred revascularization method for patients with acute coronary syndrome (ACS) $[1,2]$. Compared with bare-metal stents [3,4] and early-generation thick-strut polymer-based drug-eluting stents (DES) [5,6], newergeneration DES with thinner strut stent platforms and biocompatible durable or biodegradable polymer coatings have been shown to 
improve long-term safety and efficacy outcomes throughout 5 years of follow-up in patients with ACS. Vessel healing at culprit sites after DES implantation is however substantially delayed in patients with ACS as compared to those with chronic coronary syndrome (CCS) $[7,8]$ and is associated with a long-term risk for recurrent stent-related adverse events [9].

Newest-generation DES combining thinner-strut cobalt chromium metallic stent platforms with biodegradable polymers have been introduced to mitigate chronic inflammation and arterial injury, promote rapid endothelization and improve clinical outcomes following $\mathrm{PCI}$ compared with contemporary second-generation durable polymer DES. These features might be particularly important in the enhanced thrombotic and inflammatory environment of ACS [10] and translate into differential clinical outcomes between different DES technologies. Thin-strut biodegradable polymer sirolimus-eluting stents (BP-SES) have been shown to reduce target lesion failure (TLF) at one-year follow-up compared with durable polymer everolimus-eluting stents (DP-EES) among ACS patients with [11] and without [12] ST-segment elevation myocardial infarction (STEMI). It remains however uncertain whether the clinical benefits of BP-SES over DP-EES persist after complete degradation of the polymer coating. We therefore performed a subgroup analysis of the BIOSCIENCE trial to assess the long-term clinical outcomes with BP-SES compared to DP-EES in ACS patients.

\section{Methods}

\subsection{Study design and study population}

We performed a post-hoc subgroup analysis of patients with ACS enrolled into the BIOSCIENCE trial, an investigator-initiated, prospective, multicenter, assessor-blind, randomized, non-inferiority study that compared BP-SES with DP-EES in all-comer patients undergoing PCI. Detailed descriptions of the study rationale, design, inclusion and exclusion criteria, methods, and data management have been previously reported [13]. Briefly, CCS or ACS patients with at least one $>50 \%$ diameter de novo stenotic or in-stent restenotic lesion in a native coronary artery or a bypass graft suitable for stent implantation were randomly assigned in a 1:1 ratio to treatment with BP-SES or DP-EES. The study complied with the Declaration of Helsinki and was approved by the institutional ethics committees at all participating sites. All patients provided written informed consent for participation. The trial is registered with ClinicalTrials.gov number, NCT01443104. The final 5-year results of the BIOSCIENCE trial have been previously reported [14].

\subsection{Study procedures}

The experimental BP-SES (Orsiro, Biotronik AG, Bülach, Switzerland) is a cobalt-chromium alloy stent platform with a strut thickness of $60 \mu \mathrm{m}$ for stent diameters $\leq 3.0 \mathrm{~mm}$ and $80 \mu \mathrm{m}$ for stent diameters $>3.0 \mathrm{~mm}$, coated with an amorphous silicon carbide surface layer and covered by a poly-L-lactic acid polymer with asymmetrical maximal thickness of $7.5 \mu \mathrm{m}$ on the abluminal and $3.5 \mu \mathrm{m}$ on the luminal stent surfaces [15]. The biodegradable polymer matrix releases sirolimus at a concentration of $1.4 \mu \mathrm{g} / \mathrm{mm}^{2}$ of stent surface over a period of 12-14 weeks and degrades completely within approximatively $12--$ 24 months [15]. The comparator DP-EES (Xience Prime/Xpedition, Abbott Vascular, Abbott Park, IL, USA) is a thin-strut $(81 \mu \mathrm{m})$ cobalt-chromium alloy stent platform coated with a $7.6 \mu \mathrm{m}$ permanent vinylidene-fluoride hexafluoropropylene co-polymer, which releases with everolimus at a dose of $1.00 \mu \mathrm{g} / \mathrm{mm}^{2}$ of stent surface within 4 months.

A web-based system was used for randomization, which was stratified according to study center and presence or absence of STEMI. PCI was performed according to local guidelines at the time of enrolment. Unfractionated heparin (5000 IU or 70-100 IU/kg of body weight) or bivalirudin were administered during the procedure and the use of glycoprotein IIb/IIIa inhibitors was allowed at the discretion of the operator. Dual antiplatelet therapy combining acetylsalicylic acid (>250 mg) with clopidogrel (loading dose, $600 \mathrm{mg}$; maintenance dose, $75 \mathrm{mg}$ QD), prasugrel (loading dose, $60 \mathrm{mg}$; maintenance dose, $10 \mathrm{mg} \mathrm{QD}$ ), or ticagrelor (loading dose, $180 \mathrm{mg}$; maintenance dose, $90 \mathrm{mg}$ BID) was started before, or at the time of, $\mathrm{PCI}$ and prescribed for a recommended duration of 12 months.

\subsection{Study definitions and endpoints}

ACS was defined according to clinical manifestation, electrocardiography and cardiac biomarkers and included unstable angina, non-ST-segment elevation myocardial infarction (NSTEMI) and STEMI. The primary endpoint was target lesion failure (TLF), a composite of cardiac death, target-vessel myocardial infarction (MI), or clinically indicated target lesion revascularization (TLR), within 5 years. A clinical events committee blinded to treatment assignment independently adjudicated all adverse events. The definitions of all primary and secondary endpoints are detailed in the Supplementary Appendix.

\subsection{Statistical analyses}

Clinical outcomes were compared according to clinical presentation (ACS versus CCS) and stent type (BP-SES versus DP-EES). All analyses were performed according to the intention-to-treat principle. The results are presented as percentages for categorical variables and analyzed using the chi-square test or the Fisher's exact test, as appropriate. Continuous normally distributed variables are expressed as means \pm standard deviations and compared using the two-tailed Student's $t$-test. We used the Mantel-Cox method to calculate rate ratios (RR) with 95\% confidence intervals $(\mathrm{CI})$ and $p$ values were calculated with the log-rank test. We used time to first event for each outcome, and report numbers of patients and Kaplan-Meier estimates of cumulative incidence. We used approximate Mantel-Haenszel $\chi^{2}$ tests for effect modification to identify interactions between patient subgroups in the effect size. A pvalue of 0.05 was considered as statistically significant. SPSS software (version 24.0.0, SPSS Inc., Chicago, Illinois, USA) was used for all statistical analyses.

\section{Results}

Between March 1, 2012 and May 31, 2013, 2119 patients (3139 lesions) were enrolled in the BIOSCIENCE trial, of which 1063 (577 ACS and 486 CCS) patients were randomly allocated to treatment with BPSES and 1056 (554 ACS and 502 CCS) patients were randomly allocated to treatment with DP-EES (Fig. 1). At 5 years, follow-up data were available for 2008 (94.8\%) patients (Fig. 1). Baseline clinical characteristics stratified by presence or absence of ACS are summarized in Table 1. The mean age was $64.6 \pm 12.3$ and $64.8 \pm 12.3$ years among ACS patients treated with BP-SES and DP-EES, respectively. In the ACS subgroup, diabetes mellitus was present in $114(19.8 \%)$ patients treated with BP-SES and in 103 (18.6\%) patients treated with DP-EES. Among ACS patients, 287 (49.7\%) patients treated with BP-SES and 284 (51.3\%) patients treated with DP-EES presented with NSTEMI, whereas 212 (36.7\%) patients receiving BP-SES and 196 (35.4\%) patients receiving DP-EES presented with STEMI. At 5-year follow-up, 83 (14.6\%) patients treated with BP-SES and 86 (15.8\%) patients treated with DP-EES were on dual antiplatelet therapy in the ACS subgroup. Baseline angiographic and procedural characteristics are summarized in Supplementary Table 1. There were no significant differences in target lesion characteristics among ACS patients treated with BP-SES and DPEES. In the ACS subgroup, thrombus aspiration was performed similarly in 145 (17.3\%) patients treated with BP-SES and in 124 (15.7\%) patients receiving DP-EES.

The 5-year cumulative incidence of TLF (16.5\% vs. 22.9\%; RR, 0.69; $95 \% \mathrm{CI}, 0.57-0.85 ; p<0.001$ ) was significantly lower among patients 


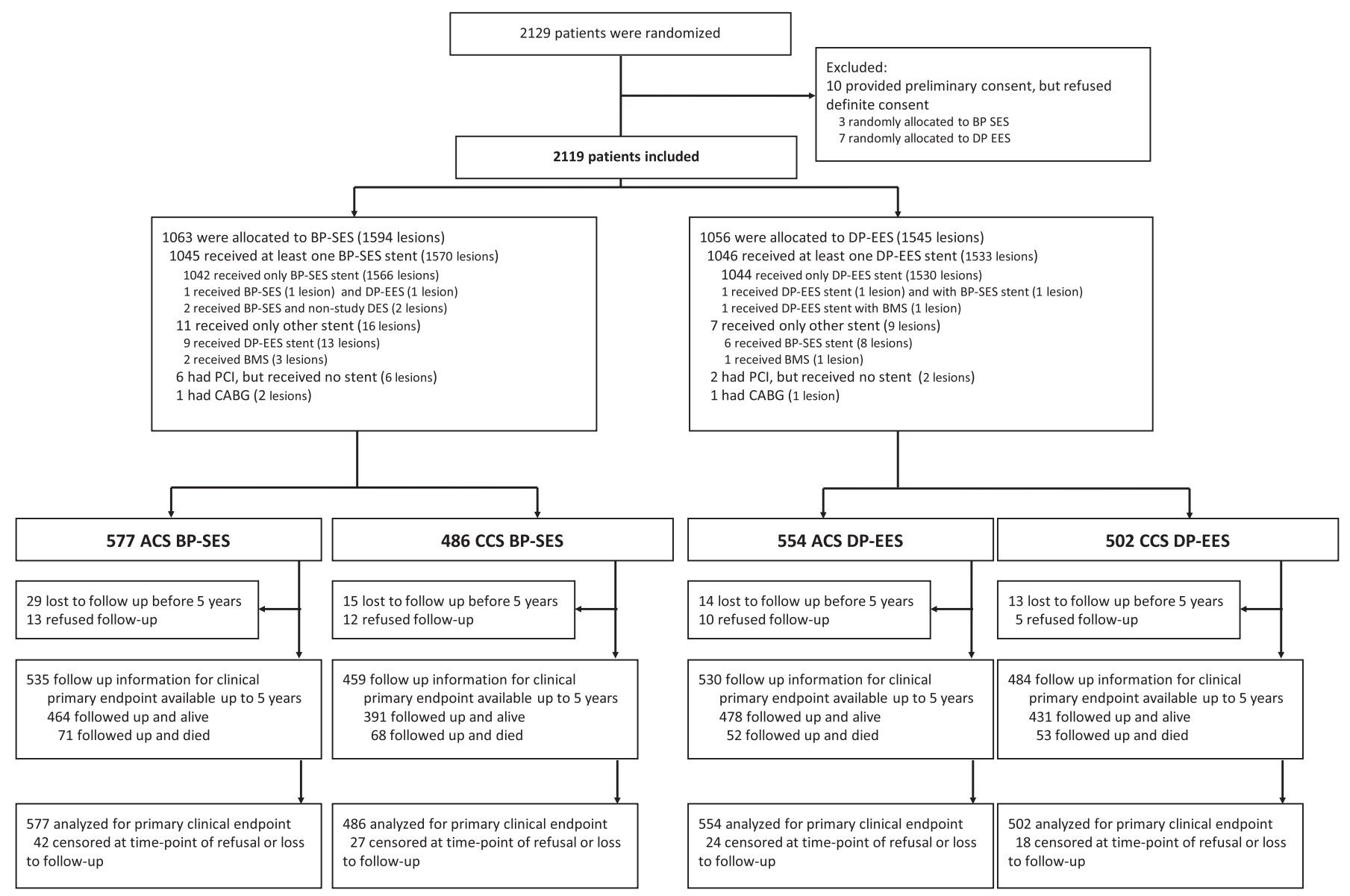

Fig. 1. Patient flow chart according to the CONSORT statement.

with ACS as compared to those with CCS. The difference was mainly driven by lower rates of target-vessel MI (5.5\% vs. 8.1\%; RR, 0.66; $95 \%$ CI, $0.47-0.94 ; p=0.019)$ and clinically indicated TLR (8.6\% vs. $12.4 \%$; $\mathrm{RR}, 0.68 ; 95 \% \mathrm{CI}, 0.51-0.90 ; p=0.006$ ) at 5 years among ACS patients compared with patients with CCS, whereas the risk of stent thrombosis was similar between groups (Supplementary Table 2). In a landmark analysis, the incidence of TLF remained significantly lower in patients with ACS compared to those with CCS (14.9\% vs. $20.3 \%$; RR, $0.72 ; 95 \%$ $\mathrm{Cl}, 0.58-0.89 ; p=0.002$ ) beyond 30 days of index revascularization throughout 5 years, a difference driven by a lower risk of clinically indicated TLR (8.4\% vs. 11.6\%; RR, 0.72; 95\% CI, 0.54-0.96; $p=0.024$ ), whereas rates of MI and stent thrombosis beyond 30 days did not differ between ACS and CCS groups (Supplementary Table 3).

At 5 years, TLF occurred in 89 patients (cumulative incidence, $16.9 \%$ ) treated with BP-SES and in 85 patients (16.0\%) treated with DP-EES (RR, $1.04 ; 95 \% \mathrm{CI}, 0.78-1.41 ; p=0.78$ ) in the ACS group, and in 109 patients (24.1\%) treated with BP-SES and 104 patients $(21.8 \%)$ treated with DPEES (RR, $1.11 ; 95 \% \mathrm{Cl}, 0.85-1.45 ; p=0.46$ ) in the CCS group ( $\mathrm{p}$ for interaction $=0.77$ ) (Table 2, Fig. 2). The cumulative incidences of cardiac death $(8.0 \%$ vs. $7.0 \%$; $p=0.66)$, target-vessel MI ( $5.2 \%$ vs. $5.8 \%$; $p=$ $0.66)$ and clinically indicated TLR $(8.9 \%$ vs. $8.3 \% ; p=0.63)$ at 5 years were similar among ACS patients treated with BP-SES or DP-EES (Table 2, Fig. 2). The cumulative 5 -year incidences of definite (1.4\% vs. $1.0 \% ; p=0.57)$ and definite or probable (5.7\% vs. $6.8 \% ; p=0.42)$ stent thrombosis did not differ between ACS patients treated with BPSES or DP-EES (Table 2). In a landmark analysis, the incidence of TLF and its individual components did not differ between 1 and 5 years in patients with ACS and CCS treated with BP-SES or DP-EES (Supplementary Table 4).

\section{Discussion}

In a post-hoc subgroup analysis of the BIOSCIENCE trial, we found a consistent treatment effect between BP-SES and DP-EES with respect to the composite endpoint of TLF at 5 years of follow-up among ACS and CCS patients. In patients with ACS, BP-SES did not confer additional long-term clinical benefits compared to DP-EES at 5 years. To our knowledge, the present study is the first to assess the long-term effects of thin-strut biodegradable polymer DES in patients with ACS versus CCS and represents the first head-to-head randomized comparison of BP-SES versus DP-EES in ACS patients throughout 5 years.

Consistent with recent evidence [16], the present analysis demonstrates that, despite most recent improvements in DES technology including thinner-strut stent platforms and biodegradable polymers, stent-related adverse outcomes continue to accrue up to 5 years after $\mathrm{PCI}$ with no evidence of plateauing. In our study, the 5-year TLF rates following PCI with newer-generation DES were however higher than those observed in a patient-level meta-analysis including $>13,000 \mathrm{pa}-$ tients treated with second-generation DES [16]. Several factors other than the DES type may impact on event rates reported across different trials. Differences in TLF rates need therefore to be interpreted taking into account patient and lesion complexity, medical therapy, functional assessment for myocardial ischemia, endpoint definitions, event reporting, data monitoring and event adjudication, all of which could have contributed to an overestimation of the 5-year incidence of TLF in our study.

We observed a lower event rates at 5 years of follow-up after treatment with newer-generation DES in patients with ACS as compared to those with CCS. The difference was primarily caused by higher rates of 
Table 1

Baseline clinical characteristics.

\begin{tabular}{|c|c|c|c|c|c|c|}
\hline \multirow[b]{3}{*}{ Patients - no. } & \multicolumn{3}{|c|}{ Acute coronary syndrome } & \multicolumn{3}{|c|}{ Chronic coronary syndrome } \\
\hline & \multirow{2}{*}{$\frac{\text { All stents }}{n=1131}$} & \multirow{2}{*}{$\frac{\text { BP-SES }}{n=577}$} & \multirow{2}{*}{$\frac{\text { DP-EES }}{n=554}$} & \multirow{2}{*}{$\begin{array}{l}\text { All stents } \\
n=988\end{array}$} & \multirow{2}{*}{$\frac{\text { BP-SES }}{n=486}$} & \multirow{2}{*}{$\begin{array}{l}\text { DP-EES } \\
n=502\end{array}$} \\
\hline & & & & & & \\
\hline Age - years (SD) & $64.7 \pm 12.3$ & $64.6 \pm 12.3$ & $64.8 \pm 12.3$ & $67.6 \pm 10.3$ & $67.9 \pm 10.4$ & $67.2 \pm 10.1$ \\
\hline Male gender - no. (\%) & $877(77.5 \%)$ & $449(77.8 \%)$ & $428(77.3 \%)$ & $755(76.4 \%)$ & $369(75.9 \%)$ & $386(76.9 \%)$ \\
\hline Body mass index $-\mathrm{kg} / \mathrm{m}^{2}$ & $27.6 \pm 4.7$ & $27.6 \pm 4.7$ & $27.5 \pm 4.7$ & $27.8 \pm 4.3$ & $27.9 \pm 4.3$ & $27.6 \pm 4.4$ \\
\hline Diabetes mellitus - no. (\%) & 217 (19.2\%) & $114(19.8 \%)$ & $103(18.6 \%)$ & $269(27.2 \%)$ & $143(29.4 \%)$ & $126(25.1 \%)$ \\
\hline Orally treated & $152(13.4 \%)$ & $82(14.2 \%)$ & $70(12.6 \%)$ & $193(19.5 \%)$ & $97(20.0 \%)$ & $96(19.1 \%)$ \\
\hline Insulin-treated & $78(6.9 \%)$ & $41(7.1 \%)$ & $37(6.7 \%)$ & $83(8.4 \%)$ & $49(10.1 \%)$ & $34(6.8 \%)$ \\
\hline Hypertension - no. (\%) & $693(61.4 \%)$ & $356(61.8 \%)$ & $337(60.9 \%)$ & $741(75.0 \%)$ & $372(76.5 \%)$ & $369(73.5 \%)$ \\
\hline Hypercholesterolemia - no. (\%) & $676(59.8 \%)$ & $351(60.9 \%)$ & $325(58.7 \%)$ & $752(76.1 \%)$ & $361(74.3 \%)$ & 391 (77.9\%) \\
\hline Current smoker - no. (\%) & $388(34.4 \%)$ & $203(35.2 \%)$ & $185(33.5 \%)$ & $221(22.4 \%)$ & $106(21.9 \%)$ & $115(22.9 \%)$ \\
\hline Family history of CAD - no. (\%) & $277(24.6 \%)$ & $139(24.2 \%)$ & $138(24.9 \%)$ & $310(31.5 \%)$ & $153(31.6 \%)$ & $157(31.4 \%)$ \\
\hline Previous MI - no. (\%) & $154(13.6 \%)$ & $88(15.3 \%)$ & $66(11.9 \%)$ & $273(27.6 \%)$ & $135(27.8 \%)$ & $138(27.5 \%)$ \\
\hline Previous PCI - no. (\%) & $197(17.4 \%)$ & $117(20.3 \%)$ & $80(14.4 \%)$ & $420(42.5 \%)$ & $208(42.8 \%)$ & $212(42.2 \%)$ \\
\hline Previous CABG - no. (\%) & $69(6.1 \%)$ & $39(6.8 \%)$ & $30(5.4 \%)$ & $142(14.4 \%)$ & $74(15.2 \%)$ & $68(13.5 \%)$ \\
\hline Atrial fibrillation - no. (\%) & 55 (4.9\%) & $27(4.7 \%)$ & $28(5.1 \%)$ & $108(10.9 \%)$ & $56(11.5 \%)$ & $52(10.4 \%)$ \\
\hline Previous stroke or TIA - no. (\%) & $39(3.4 \%)$ & $14(2.4 \%)$ & $25(4.5 \%)$ & $57(5.8 \%)$ & $25(5.1 \%)$ & $32(6.4 \%)$ \\
\hline Peripheral vascular disease - no. (\%) & $62(5.5 \%)$ & $29(5.0 \%)$ & $33(6.0 \%)$ & $114(11.5 \%)$ & $66(13.6 \%)$ & $48(9.6 \%)$ \\
\hline Renal failure (GFR $<60 \mathrm{ml} / \mathrm{min})$ - no. (\%) & $127(12.0 \%)^{\mathrm{a}}$ & $68(12.5 \%)^{\mathrm{b}}$ & $59(11.4 \%)^{\mathrm{c}}$ & $155(16.4 \%)^{d}$ & $83(17.8 \%)^{\mathrm{e}}$ & $72(15.1 \%)^{\mathrm{f}}$ \\
\hline Left ventricular ejection fraction - \% & $53.2 \pm 12.0^{\mathrm{g}}$ & $52.8 \pm 11.8^{\mathrm{h}}$ & $53.6 \pm 12.2^{\mathrm{i}}$ & $58.7 \pm 12.1^{\mathrm{j}}$ & $58.9 \pm 11.7^{\mathrm{k}}$ & $58.4 \pm 12.5^{1}$ \\
\hline \multicolumn{7}{|l|}{ Clinical presentation - no. (\%) } \\
\hline Unstable angina & $152(13.4 \%)$ & $78(13.5 \%)$ & $74(13.4 \%)$ & - & - & - \\
\hline NSTEMI & $571(50.5 \%)$ & $287(49.7 \%)$ & $284(51.3 \%)$ & - & - & - \\
\hline STEMI & $408(36.1 \%)$ & $212(36.7 \%)$ & $196(35.4 \%)$ & - & - & - \\
\hline Stable angina & - & - & - & $761(77.1 \%)$ & $371(76.5 \%)$ & $390(77.7 \%)$ \\
\hline Silent ischemia & - & - & - & $226(22.9 \%)$ & $114(23.5 \%)$ & $112(22.3 \%)$ \\
\hline \multicolumn{7}{|l|}{ Baseline medication - no. (\%) } \\
\hline Aspirin & $442(39.8 \%)^{\mathrm{m}}$ & 226 (39.8\%) & $216(39.8 \%)^{\mathrm{b}}$ & $796(81.2 \%)$ & $385(80.0 \%)$ & $411(82.4 \%)$ \\
\hline Clopidogrel & $116(10.4 \%)^{\mathrm{m}}$ & $62(10.9 \%)$ & $54(9.9 \%)^{\mathrm{b}}$ & $172(17.6 \%)$ & $67(13.9 \%)$ & $105(21.0 \%)$ \\
\hline Prasugrel & $24(2.2 \%)^{\mathrm{m}}$ & $10(1.8 \%)$ & $14(2.6 \%)^{b}$ & $56(5.7 \%)$ & $33(6.9 \%)$ & $23(4.6 \%)$ \\
\hline Ticagrelor & $55(5.0 \%)^{\mathrm{m}}$ & $22(3.9 \%)$ & $33(6.1 \%)^{b}$ & $34(3.5 \%)$ & $16(3.3 \%)$ & $18(3.6 \%)$ \\
\hline Any dual antiplatelet therapy & $169(15.2 \%)^{\mathrm{m}}$ & $83(14.6 \%)$ & $86(15.8 \%)^{\mathrm{b}}$ & $227(23.2 \%)$ & $98(20.4 \%)$ & $129(25.9 \%)$ \\
\hline Vitamin K oral anticoagulant & $43(3.9 \%)^{\mathrm{m}}$ & $23(4.0 \%)$ & $20(3.7 \%)^{b}$ & $93(9.5 \%)$ & $50(10.4 \%)$ & $43(8.6 \%)$ \\
\hline Non-vitamin $\mathrm{K}$ oral anticoagulant & $1(0.1 \%)^{\mathrm{m}}$ & $0(0.0 \%)$ & $1(0.2 \%)^{b}$ & $5(0.5 \%)$ & $3(0.6 \%)$ & $2(0.4 \%)$ \\
\hline Any anticoagulant therapy & $44(4.0 \%)^{\mathrm{m}}$ & $23(4.0 \%)$ & $21(3.9 \%)^{b}$ & $98(10.0 \%)$ & $53(11.0 \%)$ & $45(9.0 \%)$ \\
\hline Statins & $398(35.9 \%)^{\mathrm{m}}$ & $208(36.7 \%)$ & $190(35.0 \%)^{\mathrm{b}}$ & $727(74.3 \%)$ & $354(73.6 \%)$ & $373(74.9 \%)$ \\
\hline ACE inhibitors or ARB & $224(20.2 \%)^{\mathrm{m}}$ & $116(20.5 \%)$ & $108(19.9 \%)^{\mathrm{b}}$ & $324(33.1 \%)$ & $155(32.2 \%)$ & 169 (33.9\%) \\
\hline Betablockers & $346(31.2 \%)^{\mathrm{m}}$ & $183(32.3 \%)$ & $163(30.0 \%)^{b}$ & $623(63.6 \%)$ & $313(65.1 \%)$ & $310(62.2 \%)$ \\
\hline
\end{tabular}

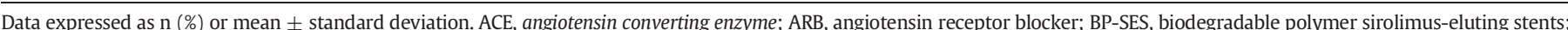

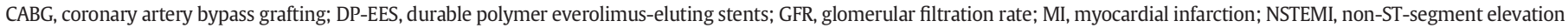
myocardial infarction; PCI, percutaneous coronary intervention; STEMI, ST-segment elevation myocardial infarction; TIA, transient ischemic attack.

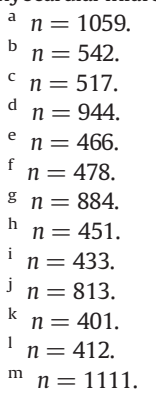

periprocedural target-vessel MI and ischemia-driven TLR in patients with CCS, which was sustained during the entire time of follow-up. These findings are consistent with previous studies indicating a higher risk of ischemic outcomes among patients with CCS as compared to ACS patients during short- and long-term follow-up [17,18]. Several reasons may account for this observation. First, even though the BIOSCIENCE trial was designed to include an all-comers patient population, only stable subjects may have been consented for participation due to the absence of consent-by-proxy directives, hence introducing a selection bias. Second, ascertainment of periprocedural MI can be challenging in patients presenting with ACS at baseline due to the elevated preprocedural cardiac biomarkers [19] and may introduce a risk of underreporting in the ACS cohort. For this reason, we performed a landmark analysis demonstrating no significant differences in MI rates between ACS and CCS patients beyond the periprocedural period throughout 5 years of follow-up. Finally, ACS and CCS patients differ with regards to baseline clinical and procedural characteristics, thus indicating a greater atherosclerotic burden and more advanced CAD in patients with CCS as compared to those with ACS. In comparison to patients with ACS, CCS patients were older and had a greater cardiovascular risk profile, as suggested by a higher prevalence of diabetes, hypertension, hypercholesterolemia, peripheral artery disease, chronic renal failure, and prior cardiovascular disease or coronary revascularization procedures. In addition, the higher prevalence of complex lesions, such as multivessel disease, chronic total occlusions, venous bypass graft disease and in-stent restenosis, in the CCS group indicates that complex coronary interventions were more frequently performed in CCS than in ACS patients. These differences may potentially explain 
Table 2

Clinical outcomes in patients with acute versus chronic coronary syndrome at 5 years.

\begin{tabular}{|c|c|c|c|c|c|c|c|c|c|}
\hline \multirow[b]{3}{*}{ Patients - no. } & \multicolumn{4}{|c|}{ Acute coronary syndrome } & \multicolumn{4}{|c|}{ Chronic coronary syndrome } & \multirow{3}{*}{$\begin{array}{l}\mathrm{p} \text {-Value for } \\
\text { interaction }\end{array}$} \\
\hline & \multirow{2}{*}{$\frac{\text { BP-SES }}{n=577}$} & \multirow{2}{*}{$\frac{\text { DP-EES }}{\mathrm{n}=554}$} & \multirow{2}{*}{$\begin{array}{l}\text { Rate ratio } \\
(95 \% \mathrm{CI})\end{array}$} & \multirow[t]{2}{*}{ p-Value } & \multirow{2}{*}{$\frac{\text { BP-SES }}{\mathrm{n}=486}$} & \multirow{2}{*}{$\frac{\text { DP-EES }}{n=502}$} & \multirow{2}{*}{$\begin{array}{l}\text { Rate ratio } \\
(95 \% \mathrm{CI})\end{array}$} & \multirow[t]{2}{*}{ p-Value } & \\
\hline & & & & & & & & & \\
\hline Target lesion failure ${ }^{a}$ & 89 (16.9) & $85(16.0)$ & $\begin{array}{l}1.04 \\
(0.78-1.41)\end{array}$ & 0.779 & $\begin{array}{l}109 \\
(24.1)\end{array}$ & $\begin{array}{l}104 \\
(21.8)\end{array}$ & $\begin{array}{l}1.11 \\
(0.85-1.45)\end{array}$ & 0.460 & 0.774 \\
\hline All-cause death & $71(13.3)$ & $52(9.7)$ & $\begin{array}{l}1.36 \\
(0.95-1.95)\end{array}$ & 0.089 & $68(15.1)$ & $53(10.9)$ & $\begin{array}{l}1.36 \\
(0.95-1.95)\end{array}$ & 0.092 & 0.993 \\
\hline Cardiac death & $41(8.0)$ & $37(7.0)$ & $\begin{array}{l}1.10 \\
(0.71-1.72)\end{array}$ & 0.664 & $40(9.1)$ & $39(8.1)$ & $\begin{array}{l}1.09 \\
(0.70-1.69)\end{array}$ & 0.707 & 0.965 \\
\hline Myocardial infarction & 49 (9.9) & $58(11.5)$ & $\begin{array}{l}0.83 \\
(0.57-1.21)\end{array}$ & 0.334 & $50(11.1)$ & $60(13.2)$ & $\begin{array}{l}0.87 \\
(0.60-1.27)\end{array}$ & 0.472 & 0.857 \\
\hline Q-wave myocardial infarction & $17(3.9)$ & $13(2.9)$ & $\begin{array}{l}1.30 \\
(0.63-2.68)\end{array}$ & 0.473 & $15(3.4)$ & $11(2.6)$ & $\begin{array}{l}1.45 \\
(0.67-3.16)\end{array}$ & 0.347 & 0.842 \\
\hline Non Q-wave myocardial infarction & $36(6.9)$ & $48(9.2)$ & $\begin{array}{l}0.74 \\
(0.48-1.14)\end{array}$ & 0.169 & $36(7.9)$ & $49(10.6)$ & $\begin{array}{l}0.76 \\
(0.50-1.18)\end{array}$ & 0.219 & 0.917 \\
\hline Target-vessel myocardial infarction & $27(5.2)$ & $30(5.8)$ & $\begin{array}{l}0.89 \\
(0.53-1.50)\end{array}$ & 0.657 & $35(7.6)$ & $39(8.5)$ & $\begin{array}{l}0.94 \\
(0.59-1.49)\end{array}$ & 0.793 & 0.874 \\
\hline Target-vessel Q-wave myocardial infarction & $11(2.1)$ & $8(1.5)$ & $\begin{array}{l}1.37 \\
(0.55-3.40)\end{array}$ & 0.501 & $10(2.2)$ & $6(1.3)$ & $\begin{array}{l}1.76 \\
(0.64-4.84)\end{array}$ & 0.269 & 0.717 \\
\hline Target-vessel non Q-wave myocardial infarction & $17(3.3)$ & $23(4.4)$ & $\begin{array}{l}0.73 \\
(0.39-1.37)\end{array}$ & 0.324 & $25(5.4)$ & $33(7.2)$ & $\begin{array}{l}0.79 \\
(0.47-1.33)\end{array}$ & 0.373 & 0.853 \\
\hline Cardiac death or any myocardial infarction & $81(15.5)$ & $88(16.8)$ & $\begin{array}{l}0.90 \\
(0.67-1.22)\end{array}$ & 0.513 & $87(19.2)$ & $91(19.3)$ & $\begin{array}{l}1.00 \\
(0.75-1.34)\end{array}$ & 0.993 & 0.635 \\
\hline Repeat revascularization (any) & $84(16.0)$ & $95(18.3)$ & $\begin{array}{l}0.88 \\
(0.66-1.19)\end{array}$ & 0.408 & $\begin{array}{l}104 \\
(23.2)\end{array}$ & $\begin{array}{l}100 \\
(21.8)\end{array}$ & $\begin{array}{l}1.12 \\
(0.85-1.48)\end{array}$ & 0.405 & 0.241 \\
\hline PCI & $81(15.4)$ & $89(17.2)$ & $\begin{array}{l}0.91 \\
(0.67-1.23)\end{array}$ & 0.545 & $96(21.3)$ & $96(20.9)$ & $\begin{array}{l}1.08 \\
(0.81-1.43)\end{array}$ & 0.606 & 0.427 \\
\hline CABG & $6(1.2)$ & $12(2.4)$ & $\begin{array}{l}0.50 \\
(0.19-1.33)\end{array}$ & 0.154 & $11(2.6)$ & $10(2.3)$ & $\begin{array}{l}1.17 \\
(0.50-2.75)\end{array}$ & 0.719 & 0.194 \\
\hline Target lesion revascularization (any) & $49(9.5)$ & $46(9.0)$ & $\begin{array}{l}1.07 \\
(0.72-1.60)\end{array}$ & 0.742 & $61(13.8)$ & $60(12.9)$ & $\begin{array}{l}1.08 \\
(0.76-1.55)\end{array}$ & 0.664 & 0.967 \\
\hline Clinically indicated target lesion revascularization & $46(8.9)$ & $42(8.3)$ & $\begin{array}{l}1.11 \\
(0.73-1.69)\end{array}$ & 0.631 & $57(12.9)$ & $55(11.8)$ & $\begin{array}{l}1.10 \\
(0.76-1.59)\end{array}$ & 0.612 & 0.981 \\
\hline PCI & $44(8.5)$ & $36(7.1)$ & $\begin{array}{l}1.24 \\
(0.80-1.92)\end{array}$ & 0.343 & $50(11.3)$ & $51(10.9)$ & $\begin{array}{l}1.04 \\
(0.70-1.53)\end{array}$ & 0.849 & 0.560 \\
\hline CABG & $4(0.8)$ & $7(1.4)$ & $\begin{array}{l}0.57 \\
(0.17-1.96)\end{array}$ & 0.368 & $8(1.9)$ & $7(1.5)$ & $\begin{array}{l}1.22 \\
(0.44-3.35)\end{array}$ & 0.705 & 0.351 \\
\hline Target-vessel revascularization (any) & $62(11.9)$ & $60(11.7)$ & $\begin{array}{l}1.04 \\
(0.73-1.48)\end{array}$ & 0.837 & $68(15.3)$ & $72(15.5)$ & $\begin{array}{l}1.00 \\
(0.72-1.40)\end{array}$ & 0.987 & 0.889 \\
\hline PCI & $59(11.3)$ & $54(10.5)$ & $\begin{array}{l}1.10 \\
(0.76-1.59)\end{array}$ & 0.616 & $61(13.7)$ & $68(14.6)$ & $\begin{array}{l}0.95 \\
(0.67-1.34)\end{array}$ & 0.774 & 0.574 \\
\hline CABG & $5(1.0)$ & $8(1.6)$ & $\begin{array}{l}0.63 \\
(0.20-1.91)\end{array}$ & 0.406 & $9(2.1)$ & $8(1.8)$ & $\begin{array}{l}1.20 \\
(0.46-3.10)\end{array}$ & 0.713 & 0.385 \\
\hline Clinically indicated target-vessel revascularization & $60(11.5)$ & $56(11.0)$ & $\begin{array}{l}1.08 \\
(0.75-1.56)\end{array}$ & 0.662 & $65(14.7)$ & $67(14.4)$ & $\begin{array}{l}1.03 \\
(0.73-1.45)\end{array}$ & 0.874 & 0.833 \\
\hline PCI & $58(11.1)$ & $51(10.0)$ & $\begin{array}{l}1.15 \\
(0.79-1.68)\end{array}$ & 0.463 & $58(13.0)$ & $64(13.7)$ & $\begin{array}{l}0.96 \\
(0.67-1.37)\end{array}$ & 0.812 & 0.485 \\
\hline CABG & $4(0.8)$ & $7(1.4)$ & $\begin{array}{l}0.57 \\
(0.17-1.96)\end{array}$ & 0.368 & $8(1.9)$ & $7(1.5)$ & $\begin{array}{l}1.22 \\
(0.44-3.35)\end{array}$ & 0.705 & 0.351 \\
\hline Target vessel failure $^{\mathrm{b}}$ & $\begin{array}{l}103 \\
(19.5)\end{array}$ & $\begin{array}{l}101 \\
(18.9)\end{array}$ & $\begin{array}{l}1.01 \\
(0.77-1.33)\end{array}$ & 0.931 & $\begin{array}{l}117 \\
(25.8)\end{array}$ & $\begin{array}{l}118 \\
(24.8)\end{array}$ & $\begin{array}{l}1.05 \\
(0.81-1.35)\end{array}$ & 0.730 & 0.864 \\
\hline $\begin{array}{l}\text { Death, myocardial infarction or any repeat } \\
\text { revascularization }{ }^{c}\end{array}$ & $\begin{array}{l}154 \\
(28.3)\end{array}$ & $\begin{array}{l}150 \\
(28.1)\end{array}$ & $\begin{array}{l}1.02 \\
(0.81-1.27)\end{array}$ & 0.882 & $\begin{array}{l}171 \\
(36.7)\end{array}$ & $\begin{array}{l}158 \\
(32.8)\end{array}$ & $\begin{array}{l}1.15 \\
(0.93-1.43)\end{array}$ & 0.207 & 0.443 \\
\hline Cerebrovascular event (any) & $20(3.9)$ & $26(5.0)$ & $\begin{array}{l}0.76 \\
(0.42-1.36)\end{array}$ & 0.355 & $17(3.8)$ & $12(2.6)$ & $\begin{array}{l}1.50 \\
(0.72-3.15)\end{array}$ & 0.277 & 0.154 \\
\hline Stroke (any) ${ }^{\mathrm{d}}$ & $15(2.9)$ & $23(4.5)$ & $\begin{array}{l}0.64 \\
(0.34-1.23)\end{array}$ & 0.182 & $12(2.7)$ & $11(2.4)$ & $\begin{array}{l}1.15 \\
(0.51-2.62)\end{array}$ & 0.733 & 0.274 \\
\hline Transient ischemic attack & $6(1.1)$ & $5(1.0)$ & $\begin{array}{l}1.18 \\
(0.36-3.89)\end{array}$ & 0.779 & $5(1.2)$ & $1(0.2)$ & $\begin{array}{l}5.31 \\
(0.63-44.92)\end{array}$ & 0.086 & - \\
\hline Definite stent thrombosis & $7(1.4)$ & $5(1.0)$ & $\begin{array}{l}1.39 \\
(0.44-4.37)\end{array}$ & 0.573 & $9(2.0)$ & $11(2.4)$ & $\begin{array}{l}0.87 \\
(0.36-2.10)\end{array}$ & 0.749 & 0.521 \\
\hline Definite or probable stent thrombosis & $30(5.7)$ & $36(6.8)$ & $\begin{array}{l}0.82 \\
(0.51-1.33)\end{array}$ & 0.421 & $32(7.0)$ & $40(8.7)$ & $\begin{array}{l}0.84 \\
(0.52-1.34)\end{array}$ & 0.457 & 0.951 \\
\hline
\end{tabular}

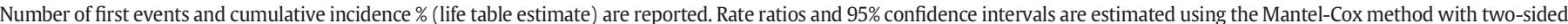

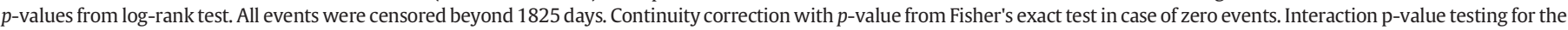

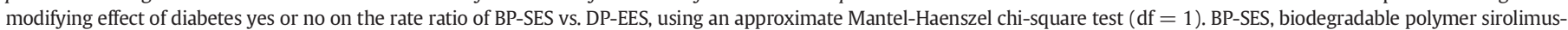
eluting stents; CABG, coronary artery bypass grafting; CI, confidence interval; DP-EES, durable polymer everolimus-eluting stents; PCI, percutaneous coronary intervention.

a Primary endpoint, defined as the composite of cardiac death, target-vessel Q-wave or non Q-wave myocardial infarction, clinically indicated target lesion revascularization.

b Defined as the composite of cardiac death, any Q-wave or non Q-wave myocardial infarction, and any target-vessel revascularization.

c Patient oriented composite endpoint.

d Includes ischemic stroke, intracerebral hemorrhagic stroke and cerebrovascular event of unclear etiology. 

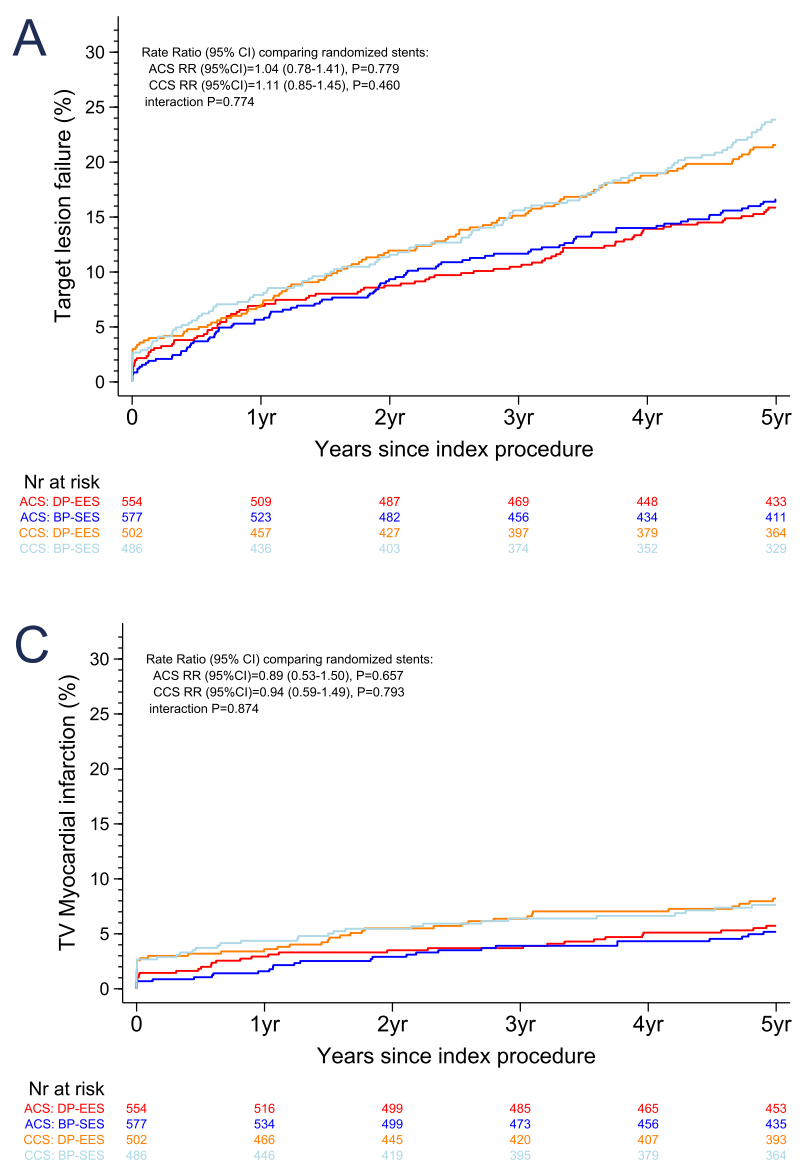
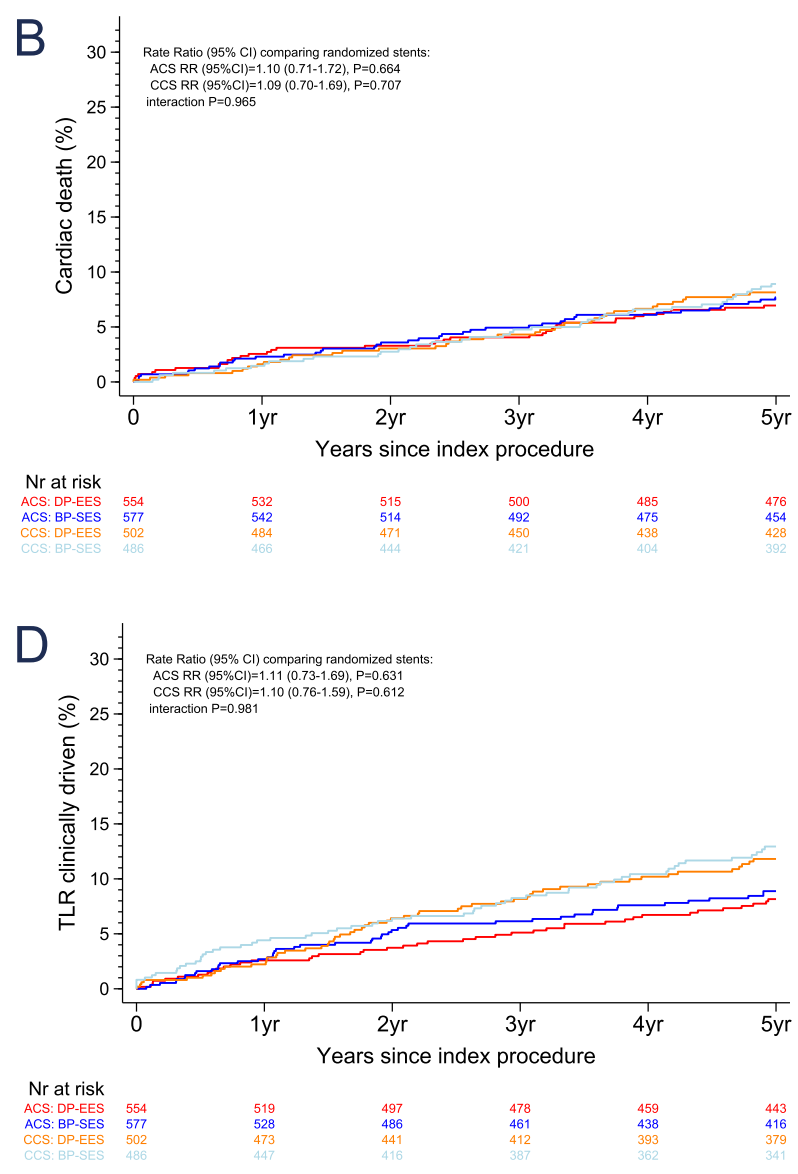

Fig. 2. Time to event curves for the composite endpoint of target lesion failure and individual components of the primary endpoint up to 5 years of follow-up.

the higher rates of repeat revascularization at five years of follow-up observed among patients with CCS as compared to those with ACS.

Newer-generation DES have been shown to improve device- and patient-oriented clinical outcomes up to 5 years of follow-up compared to bare-metal stents [3,4] and early-generation polymer-based DES [5,6] in patients with ACS, a difference mainly driven by lower rates of repeat revascularization [3-6] and target-vessel MI [4]. The prothrombotic and proinflammatory milieu of ACS contributes to delayed arterial healing at ACS culprit sites and translates into the occurrence of late stent-related adverse events with newer-generation polymer-based DES [7,9], hence disclosing the need for further refinements in DES technology. Newergeneration DES with thinner stent struts and biodegradable polymer coatings have the potential to mitigate stent-induced arterial injury and chronic permanent polymer-related vascular inflammation, faster endothelialization and improve long-term clinical outcomes compared to contemporary polymer-based DES. Beyond the potential effects of biodegradable polymers in reducing chronic inflammation [20], thinner stent strut thickness has been shown to mitigate acute stent-induced thrombogenicity [10] and reduce TLF rates, a difference mainly driven by a lower risk of MI, compared with newer-generation thicker-strut DES [21]. In particular, BP-SES were found non-inferior [14] or superior [22] to DP-EES with respect to TLF at up to 5 years of follow-up among all-comer patients. Patients with ACS may represent a particular subset which may theoretically derive greater long-term benefits from treatment with thin-strut biodegradable polymer DES technology. In the ACS subgroup analysis of the BIOFLOW-V trial [10], BP-SES were associated with lower TLF rates at one-year follow-up, a difference driven by a lower risk of periprocedural and spontaneous target-vessel myocardial MI, compared to DP-EES. The study however excluded highest-risk patients with STEMI. The absence of between-stent difference in target-vessel MI rates in our study might be explained by differential definitions between studies and the particular challenge to adjudicate periprocedural myocardial reinfarction among cardiac biomarker-positive ACS patients [19]. Among STEMI patients undergoing primary PCI, BP-SES were recently found superior to DP-EES with respect to TLF, a difference driven by a lower risk of clinically indicated TLR, at one year of follow-up [9]. In both studies, the clinical advantages of BP-SES over DP-EES among ACS patients emerged well before resorption of the biodegradable polymer and it remains uncertain whether the potential benefits might persist during long-term follow-up as complete polymer biodegradation after drug elution is expected to render the BPSES surface similar to a BMS. To our knowledge, the present analysis represents the first randomized study comparing longer-term clinical outcomes between BP-SES and DP-EES among ACS patients with and without STEMI. Our results indicate similar long-term outcomes with respect to the primary endpoint of TLF and its individual components between BP-SES and DP-EES irrespective of the baseline clinical presentation throughout 5 years of follow-up. Event rates observed in our analysis are consistent with previous studies reporting the long-term outcomes following PCI with newer-generation DES in patients with acute MI $[3,4,6]$. Noteworthy, the rates of definite stent thrombosis at 5 years were low and did not differ between treatment groups despite the inclusion of an all-comer ACS population with a high proportion of STEMI patients. Overall, these findings confirm the long-term safety and efficacy profiles of newest generation thinner-strut highly biocompatible DES with either biodegradable or thromboresistant permanent fluorinated polymer coatings for percutaneous treatment across the entire spectrum of CAD, including in patients with ACS. 


\subsection{Limitations}

The present analysis must be interpreted in view of several limitations. As per the main trial design, randomization into the BIOSCIENCE trial was not stratified according to presence or absence of ACS, but to presence or absence of STEMI. The present post-hoc subgroup analysis was not powered to assess differences in reported endpoints between the two treatment arms. The results are therefore hypothesisgenerating and must be cautiously interpreted. In addition to strut thickness and the biodegradable polymer, BP-SES and DP-EES designs also differ with respect stent platform geometry, polymer composition, thickness, distribution and time to complete degradation, and antiproliferative drug composition and elution kinetics, all of which may potentially affect long-term clinical outcomes after DES implantation.

\section{Conclusion}

In a subgroup analysis of the BIOSCIENCE trial, we found a consistent treatment effect with respect to TLF at 5 years between patients with ACS or CCS treated with BP-SES or DP-EES. Among ACS patients, there was no difference in long-term clinical outcomes between BP-SES and DP-EES throughout 5 years of follow-up.

\section{Funding source}

BIOSCIENCE was an investigator-initiated study which was supported by a dedicated research grant from Biotronik, Bülach, Switzerland. The principal investigator (SW), co-principal investigator (TP) and trial statistician (DH) are responsible for the study design and conduct, as well as all analyses. The funding source was not involved in the study design, data collection and management, and had no role in the analysis and interpretation of the study data, writing of the manuscript or decision to submit for publication.

\section{Author contributions}

TP, DH, PJ, and SW conceived of the study. TP, DH, PJ, and SW had responsibility for the design of the study. JFI, TP, DH, MR, DT, OM, MB, SC, DW, CK, FC, PJ, and SW were responsible for the acquisition of data. DH did the analysis and interpreted the results in collaboration with all other authors. JFI, TP and DH wrote the first draft of the article. All authors critically revised the report for important intellectual content and approved the final version.

\section{Declaration of competing interest}

JI reports institutional research grants from Biotronik, Abbott Vascular and Astra Zeneca, personal fees from Biotronik, Philips Volcano, Astra Zeneca, Terumo, Medtronic and Cardinal Health, outside the submitted work. DH is affiliated with Clinical Trials Unit Bern (CTU Bern), University of Bern (Bern, Switzerland), which has a staff policy of not accepting honoraria or consultancy fees. However, CTU Bern is involved in design, conduct, or analysis of clinical studies funded by not-for-profit and forprofit organisations. In particular, pharmacetical and medical device companies provide direct funding to some of these studies. For an upto-date list of CTU Bern's conflicts of interest see www.ctu.unibe.ch/ research/declaration_of_interest/index_eng.html. MR reports institutional research grants from Terumo, Boston Scientific, Medtronic, Abbott Vascular, and Biotronik, outside the submitted work. PJ serves as an unpaid member of the steering group of trials funded by AstraZeneca, Biotronik, Biosensors, St Jude Medical, and The Medicines Company, and received research grants to their institution from AstraZeneca, Biotronik, Biosensors International, Eli Lilly, and The Medicines Company, and honoraria to their institution for participation in advisory boards from Amgen, but has not received personal payments by any pharmaceutical company or device manufacturer. PJ is a Tier 1 Canada Research Chair in Clinical Epidemiology of Chronic Diseases. MV reports grants and personal fees from Abbott, Terumo, and AstraZeneca, personal fees from Bayer, Dalichi Sankyo, Amgen, Alvimedica, Idorsia, Coreflow, Vifor, Bristol-Myers Squibb, and iVascular, and grants from Medicure, ouside the submitted work. SW received research grants to his institution from Abbott, Amgen, Biotronik, Boston Scientific, Edwards Lifesciences, Medtronic, Medicines Company, and St Jude. TP received research grants to his institution from Biotronik, Boston Scientific and Edwards Lifesciences, and speaker fees from Biotronik and Boston Scientific Consultancy from HighLIFE SAife SASSAS. The other authors report no conflicts.

\section{Appendix A. Supplementary data}

Supplementary data to this article can be found online at https://doi. org/10.1016/j.carrev.2021.02.008.

\section{References}

[1] Collet JP, Thiele H, Barbato E, Barthélémy O, Bauersachs J, Bhatt DL, et al. 2020 ESC Guidelines for the management of acute coronary syndromes in patients presenting without persistent ST-segment elevation. Eur Heart J. 2021;4:1289-367.

[2] Ibanez B, James S, Agewall S, Antunes MJ, Bucciarelli-Ducci C, Bueno H, et al. 2017 ESC guidelines for the management of acute myocardial infarction in patients presenting with ST-segment elevation: the task force for the management of acute myocardial infarction in patients presenting with ST-segment elevation of the European Society of Cardiology (ESC). Eur Heart J. 2018;39:119-77.

[3] Sabaté M, Brugaletta S, Cequier A, Iiguez A, Serra A, Jiménez-Quevedo P, et al. Clinical outcomes in patients with ST-segment elevation myocardial infarction treated with everolimus-eluting stents versus bare-metal stents (EXAMINATION): 5-year results of a randomised trial. Lancet. 2016;387:357-66.

[4] Räber L, Yamaji K, Kelbæk H, Engstrøm T, Baumbach A, Roffi M, et al. Five-year clinical outcomes and intracoronary imaging findings of the COMFORTABLE AMI trial: randomized comparison of biodegradable polymer-based biolimus-eluting stents with bare-metal stents in patients with acute ST-segment elevation myocardial infarction. Eur Heart J. 2019;40:1909-19.

[5] Bangalore S, Amoroso N, Fusaro M, Kumar S, Feit F. Outcomes with various drugeluting or bare metal stents in patients with ST-segment-elevation myocardial infarction: a mixed treatment comparison analysis of trial level data from 34068 patient-years of follow-up from randomized trials. Circ Cardiovasc Interv. 2013;6: 378-90.

[6] de Waha A, King LA, Stefanini GG, Byrne RA, Serruys PW, Meier B, et al. Long-term outcomes of biodegradable versus durable polymer drug-eluting stents in patients with acute ST-segment elevation myocardial infarction: a pooled analysis of individual patient data from three randomised trials. EuroIntervention. 2015;10:1425-31.

[7] Nakazawa G, Finn AV, Joner M, Ladich E, Kutys R, Mont EK, et al. Delayed arterial healing and increased late stent thrombosis at culprit sites after drug-eluting stent placement for acute myocardial infarction patients: an autopsy study. Circulation. 2008;118:1138-45.

[8] Räber L, Zanchin T, Baumgartner S, Taniwaki M, Kalesan B, Moschovitis A, et al. Differential healing response attributed to culprit lesions of patients with acute coronary syndromes and stable coronary artery after implantation of drug-eluting stents: an optical coherence tomography study. Int J Cardiol. 2014;173:259-67.

[9] Sarno G, Lagerqvist B, Nilsson J, Frobert O, Hambraeus K, Varenhorst C, et al. Stent thrombosis in new-generation drug-eluting stents in patients with STEMI undergoing primary PCI: a report from SCAAR. J Am Coll Cardiol. 2014;64:16-24.

[10] Kolandaivelu K, Swaminathan R, Gibson WJ, Kolachalama VB, Nguyen-Ehrenreich KL, Giddings VL, et al. Stent thrombogenicity early in high-risk interventional settings is driven by stent design and deployment and protected by polymer-drug coatings. Circulation. 2011;123:1400-9.

[11] Iglesias JF, Muller O, Heg D, Roffi M, Kurz DJ, Moarof I, et al. Biodegradable polymer sirolimus-eluting stents versus durable polymer everolimus-eluting stents in patients with ST-segment elevation myocardial infarction (BIOSTEMI): a single-blind, prospective, randomised superiority trial. Lancet. 2019;394:1243-53.

[12] Roguin A, Kandzari DE, Marcusohn E, Koolen JJ, Doros G, Massaro JM, et al. Subgroup analysis comparing ultrathin, bioresorbable polymer sirolimus-eluting stents versus thin, durable polymer everolimus-eluting stents in acute coronary syndrome patients. Circ Cardiovasc Interv. 2018;11:e007331.

[13] Pilgrim T, Roffi M, Tüller D, Muller O, Vuilliomenet A, Cook S, et al. Randomized comparison of biodegradable polymer sirolimus-eluting stents versus durable polymer everolimus-eluting stents for percutaneous coronary revascularization: rationale and design of the BIOSCIENCE trial. Am Heart J. 2014;168:256-61.

[14] Pilgrim T, Piccolo R, Heg D, Roffi M, Tüller D, Muller O, et al. Ultrathin-strut, biodegradable-polymer, sirolimus-eluting stents versus thin-strut, durablepolymer, everolimus-eluting stents for percutaneous coronary revascularisation: 5-year outcomes of the BIOSCIENCE randomised trial. Lancet. 2018;392:737-46.

[15] Iglesias JF, Roffi M, Degrauwe S, Secco GG, Aminian A, Windecker S, et al. Orsiro cobalt-chromium sirolimus-eluting stent: present and future perspectives. Expert Rev Med Devices. 2017;11:773-88. 
[16] Madhavan MV, Kirtane AJ, Redfors B, Généreux P, Ben-Yehuda O, Palmerini T, et al. Stent-related adverse events $>1$ year after percutaneous coronary intervention. J Am Coll Cardiol. 2020;75:590-604.

[17] Vranckx P, Kalesan B, Stefanini GG, Farooq V, Onuma Y, Silber S, et al. Clinical outcome of patients with stable ischaemic heart disease as compared to those with acute coronary syndromes after percutaneous coronary intervention. EuroIntervention. 2015;11:171-9.

[18] Pilgrim T, Vranckx P, Valgimigli M, Stefanini GG, Piccolo R, Rat J, et al. Risk and timing of recurrent ischemic events among patients with stable ischemic heart disease, non-ST-segment elevation acute coronary syndrome, and ST-segment elevation myocardial infarction. Am Heart J. 2016;175:56-65.

[19] Vranckx P, Cutlip DE, Mehran R, Kint PP, Silber S, Windecker S, et al. Myocardial infarction adjudication in contemporary all-comer stent trials: balancing sensitivity and specificity. Addendum to the historical MI definitions used in stent studies. EuroIntervention. 2010;5:871-4.

[20] Koppara T, Joner M, Bayer G, Steigerwald K, Diener T, Wittchow E. Histopathological comparison of biodegradable polymer and permanent polymer based sirolimus eluting stents in a porcine model of coronary stent implantation. Thromb Haemost. 2012;107:1161-71.

[21] Bangalore S, Toklu B, Patel N, Feit F, Stone GW. Newer-generation ultrathin strut drug-eluting stents versus older second-generation thicker strut drug-eluting stents for coronary artery disease. Circulation. 2018;138:2216-26.

[22] Kandzari DE, Koolen JJ, Doros G, Garcia-Garcia HM, Bennett J, Roguin A, et al. Ultrathin bioresorbable polymer sirolimus-eluting stents versus thin durable polymer everolimus-eluting stents for coronary revascularization: three-year outcomes from the randomized BIOFLOW V trial. J Am Coll Cardiol Intv. 2020;13:1343-53. 